\title{
Consumo alimentar de adolescentes matriculados na rede pública de ensino de Piracicaba - SP ${ }^{1}$
}

\author{
Daniela Cristina Rossetto Caroba ${ }^{1}$, Marina Vieira da Silva ${ }^{2}$
}

\begin{abstract}
Este estudo teve por objetivo avaliar quantitativamente o consumo alimentar de uma amostra estratificada proporcional de 578 alunos (adolescentes), de ambos os gêneros, matriculados em sete escolas públicas da cidade de Piracicaba, São Paulo. As informações sobre o consumo alimentar dos alunos foram obtidas por meio do método Recordatório 24 horas. Para os cálculos do conteúdo de energia, macronutrientes (carboidratos, lipídios e proteínas), fibras, tiamina, riboflavina, niacina, folacina, vitamina E, fósforo e ferro, utilizou-se o software Virtual Nutri (PHILIPPI et al, 1996). Foram elaboradas análises de regressão múltipla, utilizando-se os recursos do programa STATISTICAL ANALYSIS SYSTEM (SAS, 1998). Entre os principais resultados, merece destaque o consumo de energia, fibras, folacina, vitamina $\mathrm{E}$ e fósforo, dos adolescentes, inferior ao preconizado. Quanto à tiamina, riboflavina e niacina, foi verificado que o consumo desses nutrientes se revelou acima do recomendado para o grupo da pesquisa. Em relação a proteínas, carboidratos, lipídios e ferro, os alunos integrantes da amostra apresentaram média de consumo que atende aos valores preconizados. Adotando-se a técnica estatística de regressão múltipla, foi possível notar que em cada mês adicional na vida dos meninos há aumento do consumo de 4,73 kcal, enquanto que, para as meninas, a ingestão diminui $2,14 \mathrm{kcal}$ por mês. Destaca-se que a fase da adolescência é o momento privilegiado para as intervenções na área da saúde e da nutrição, tendo em vista a adoção de hábitos de vida saudáveis e a promoção da saúde na vida adulta.
\end{abstract}

Palavras-chave: adolescência, consumo alimentar, hábitos alimentares.

\section{Food consumption of adolescents registered in the public schools of the city of Piracicaba - SP}

The objective of this paper was to evaluate quantitatively the food consumption of 578 adolescents, of both genders, registered at seven public schools of the city of Piracicaba, state of São Paulo. Food intake data were collected throught a 24-hour dietary recall. For the calculations of the energy content, macronutrients (carbohydrate, fat and protein), fiber, thiamin, riboflavin, niacin, folate, vitamin E, phosphorus and iron, the Virtual Nutri software (PHILIPPI et al, 1996) was used. Analyses of multiple regression were performed using the resources of the program Statistical Analysis System - SAS. The results revealed that the adolescent's food consumption was deficient in energy, dietary fiber, folate, vitamin $\mathrm{E}$ and phosphorus, while the consumption of thiamin, riboflavin and niacin were above of the recommendations for the group. With regard to protein, carbohydrate, fat and iron, the adolescents' intake was commensurate with the recommended values. Using the multiple regression statistics technique, it was possible to notice that at each additional month of the boys' life there was an addition of $4.73 \mathrm{kcal}$ to his energy intake, whereas for the girls,

\footnotetext{
${ }^{1}$ Artigo baseado na dissertação "A escola e o consumo alimentar de adolescentes matriculados na rede pública de ensino" (162p.), financiada pela FAPESP (Proc. 99/10362-8), defendida em 2002. Programa de Pós-Graduação em Ciência e Tecnologia de Alimentos da Escola Superior de Agricultura "Luiz de Queiroz" - ESALQ/USP.

${ }^{1}$ Mestre em Ciências (ESALQ/USP). Doutoranda em Nutrição Humana Aplicada - PRONUT/USP. Endereço para correspondência: Rua Regente Feijó, 314. Centro - Piracicaba - SP. CEP: 13400-100. E-mail: dcrcarob@usp.br

${ }^{2}$ Profa. Dra. do Departamento de Agroindústria, Alimentos e Nutrição, Escola Superior de Agricultura "Luiz de Queiroz" - ESALQ/ USP. E-mail: mvdsilva@esalq.usp.br
} 
there was a decrease of $2.14 \mathrm{kcal}$ per month. It can be inferred that adolescence is a prime phase in life for health and nutrition corrective interventions aiming at the adoption of healthful habits and the promotion of health later in life.

Keywords: adolescence, food consumption, food habits.

\section{Introdução}

O número de adolescentes, na maioria dos países do continente americano, tem aumentado nas últimas décadas. No Brasil, segundo dados do Instituto Brasileiro de Geografia e Estatística - IBGE (2001), os indivíduos com idade entre 10 e 17 anos representavam $17,1 \%$ da população.

À medida que o tamanho relativo da população juvenil cresce, também aumenta a necessidade de informação sobre o contexto em que o desenvolvimento social e físico ocorre, e sobre os comportamentos que podem afetar a saúde do referido grupamento.

A adolescência é uma etapa do desenvolvimento acompanhada de processos de crescimento e de maturação, tanto do ponto de vista somático como psicológico. Trata-se de um período de elevada demanda nutricional, e, por este motivo, a nutrição desempenha um papel importante no desenvolvimento do adolescente. Tal importância decorre do fato de que a adoção de dieta inadequada pode influenciar de forma desfavorável o crescimento somático (ALBANO \& SOUZA, 2001).

O envolvimento da adolescência com a nutrição constitui um aspecto de grande relevância, pois nesta fase da vida acontece o último momento de aceleração do crescimento, adquirindo o indivíduo, aproximadamente, $15 \%$ de sua estatura definitiva, $45 \%$ de sua massa esquelética máxima e $50 \%$ de seu peso adulto ideal (ALBUQUERQUE \& MONTEIRO, 2002).

Segundo FISBERG et al. (2000), o comportamento alimentar do adolescente vincula-se fortemente aos padrões manifestados pelo grupo etário a que pertence. Omissão de refeições, consumo de alimentos com elevado conteúdo energético e pobres em nutrientes, ingestão precoce de bebidas alcoólicas e tendências a restrições dietéticas são fatores que podem levar à obesidade ou à anorexia nervosa.

Diferenças entre os dois gêneros, com relação a problemas alimentares, surgem nesta fase da vida. As meninas experimentam mais conflitos, relacionados à comida, ao peso e formas do corpo, do que os meninos. As pressões para a manutenção da magreza já estão presentes, como pode ser notado ao observar as modalidades de regimes adotados por meninas, que revelam um medo mórbido de engordar (KAUFMANN, 2002).

A obesidade é considerada uma epidemia mundial, presente tanto em países desenvolvidos como em desenvolvimento. As tendências de transição nutricional ocorrida neste século em diferentes países do mundo convergem para uma dieta mais rica em gorduras (particularmente as de origem animal), açúcares e alimentos refinados, e reduzida em carboidratos complexos e fibras. Aliando esse fator ao declínio progressivo da atividade física dos indivíduos, percebem-se alterações concomitantes na composição corporal, principalmente o aumento da gordura (FRANCISCHI et al, 2000).

Os métodos de avaliação do consumo alimentar, mais freqüentemente adotados para a avaliação da situação de grupos populacionais de crianças e adolescentes, normalmente são semelhantes aos utilizados em análises envolvendo os adultos. Reconhece-se que cada um desses métodos tem suas vantagens e limitações. Atualmente, métodos como o Recordatório 24 horas, história dietética e registro de alimentos são adotados majoritariamente na avaliação dietética (CAVALCANTE et al, 2004). 
A avaliação precisa do consumo alimentar de crianças e adolescentes desperta grande preocupação, especialmente para os profissionais da área de saúde, porque a formação de hábitos alimentares inadequados pode ser considerada um potencial fator de risco para enfermidades crônicas não transmissíveis (VILLAR, 2001).

Tendo em vista o exposto, o presente trabalho tem por objetivo avaliar quantitativamente o consumo alimentar de adolescentes, de ambos os gêneros, matriculados em sete escolas públicas da cidade de Piracicaba, São Paulo.

\section{Metodologia}

A pesquisa foi desenvolvida em Piracicaba, município paulista localizado na Região Administrativa de Campinas, situado a 138,1 km da capital do Estado de São Paulo. De acordo com os dados do Censo de 2000, o município possuía $1.452 \mathrm{~km}^{2}$ de extensão territorial e 329.158 habitantes, com taxa de urbanização de 96,42\% (IBGE, 2005).

Inicialmente, foi realizado um levantamento junto à Diretoria de Ensino de Piracicaba, com o objetivo de conhecer a distribuição das escolas da Rede Estadual de Ensino, de acordo com a localização geográfica no município e o número total de alunos matriculados, no ano de 2000.

Tendo por base o conjunto das unidades de ensino, sorteou-se uma escola de cada região, com exceção da Região I, onde foram sorteadas duas unidades de ensino devido à mesma concentrar maior número de escolas. Tal procedimento contribuiu para que fosse assegurada a representatividade, na amostra, das escolas e da população de alunos matriculados. Desse modo, obteve-se uma amostra de sete escolas que integram a pesquisa (de um total de 54 unidades estaduais de ensino no município), caracterizando uma amostra estratificada proporcional de 578 adolescentes.

Com base na lista de freqüência dos professores, das unidades de ensino selecionadas, sorteou-se alternadamente $10 \%$ de escolares (da faixa etária de 11 a 16 anos) de cada Região, de maneira a obter proporções similares de adolescentes do gênero masculino e do gênero feminino e, contemplando, também, a representação do total dos estratos de idade.
As informações sobre o consumo alimentar foram obtidas, por meio da aplicação de um Recordatório 24 horas, junto aos adolescentes participantes da pesquisa.

Para os cálculos do conteúdo de energia e nutrientes da dieta dos escolares, utilizou-se o software Virtual Nutri, versão 1.0 (PHILIPPI et al, 1996). As análises estatísticas foram elaboradas utilizando-se os recursos do programa STATISTICAL ANALYSIS SYSTEM - SAS (1998).

No tocante à análise qualitativa do Valor Energético Total - VET da dieta, foram adotadas, como referência, as recomendações preconizadas pelo INSTITUTE OF MEDICINE (2002), que considera aceitáveis, para indivíduos de 4 a 18 anos, os seguintes intervalos de participação de macronutrientes: $45-65 \%$ provenientes dos carboidratos, $25-35 \%$ dos lipídios e $10-30 \%$ das proteínas.

Para energia, micronutrientes (tiamina, riboflavina, niacina, folacina, vitamina $\mathrm{E}$, fósforo $\mathrm{e}$ ferro) e fibras julgou-se pertinente adotar também as recomendações do INSTITUTE OF MEDICINE (1997, 1998, 2000, 2001 e 2002), preconizadas para indivíduos com idade entre 14 e 18 anos. Para efeitos de comparação entre os dados obtidos no presente trabalho com os valores preconizados, optou-se por utilizar a média dos valores definidos para meninos e meninas, conforme mostrado no Quadro 1.

Para analisar como o consumo de energia e nutrientes varia em função da idade e do gênero foi utilizado o seguinte modelo de regressão múltipla:

$$
Y_{i}=\alpha+\beta G_{i}+\gamma I_{i}+\delta G_{i} I_{i}+u_{i}
$$

em que $Y_{i}$ é o consumo (de energia ou de determinado nutriente, na unidade de medida adequada), $G_{i}$ é uma variável binária determinada a captar o efeito do gênero ( $G i=0$ ou $G i=1$, conforme o gênero seja masculino ou feminino, respectivamente), li é a idade (em meses) e ui é um erro aleatório com as propriedades usuais.

Note-se que o modelo inclui o efeito da interação entre idade e gênero $(G i l i)$.

O ́ndice $i$ indica o aluno $i$ (com variando de 1 a 578). 
Quadro 1. Recomendações (média) de energia e nutrientes para indivíduos de ambos gêneros, com idade entre 14 e 18 anos.

\begin{tabular}{lc}
\hline Energia e Nutrientes & Valores preconizados \\
\hline Energia (kcal) & 2493,5 \\
Tiamina (mg) & 1,1 \\
Riboflavina (mg) & 1,2 \\
Niacina (mg) & 15,0 \\
Folacina (mg) & 400,0 \\
Vitamina E (mg) & 15,0 \\
Fósforo (mg) & 1250,0 \\
Ferro (mg) & 13,0 \\
Fibras (g) & 32,0 \\
\hline
\end{tabular}

Fonte: INSTITUTE OF MEDICINE (1997, 1998, 2000, 2001 e 2002). Yié

Para os meninos, como $G i=0$, o valor esperado de

$$
\mathrm{E}(Y i)=\alpha+\gamma I i
$$

Para as meninas, como $G i=1$, o valor esperado de Yié

$$
\mathrm{E}(Y i)=\alpha+\beta+(\delta) I i
$$

Verifica-se que o parâmetro $y$ representa o aumento em $\mathrm{E}\left(\mathrm{Y}_{i}\right)$ para cada mês adicional na idade de um menino. Para as meninas, esse aumento é dado por $y+\delta$.

Note-se que $\delta$ representa a diferença de comportamento entre meninas e meninos, no que se refere ao efeito da idade no consumo esperado.

As estimativas de mínimos quadrados dos parâmetros $\alpha, \beta, \gamma$ e $\delta$ serão indicadas por $\mathbf{a}, \mathbf{b}, \mathbf{c}$ e $\mathbf{d}$.

Também foram elaboradas análises envolvendo os percentis da distribuição do consumo de energia e nutrientes selecionados, de acordo com o gênero dos alunos. Para a elaboração da totalidade das referidas análises, utilizou-se o software $S A S$ (1998).

\section{Resultado e discussão}

Foram elaboradas análises relativas ao consumo alimentar dos escolares, tendo por base os dados referentes à energia, macronutrientes (carboidratos, lipídios e proteínas), fibras, tiamina, riboflavina, niacina, folacina, vitamina $\mathrm{E}$, fósforo e ferro.

A seguir, apresenta-se a Tabela 1, que reúne os resultados referentes ao conteúdo médio diário de energia e nutrientes da dieta dos escolares.

Os resultados contidos na Tabela 1 revelam que os adolescentes apresentaram um consumo médio diário de energia de 1859,1 kcal, inferior às recomendações de energia preconizadas (cerca de $2493,5 \mathrm{kcal}$ ) para o grupo com idade entre 14 e 18 anos, de acordo com o INSTITUTE OF MEDICINE (2002). No entanto, o valor do desvio-padrão $(758,2)$ é elevado, indicando que há grande variação de consumo entre os alunos integrantes da amostra. Note-se que o valor máximo observado para o consumo energético alcança 4838,7 kcal, obviamente um valor que reflete uma ingestão substancial de energia.

Cabe registrar que a pesquisa de CAROBA (2002)*, tendo como amostra os 578 adolescentes da rede pública de ensino de Piracicaba, revelou a prevalência de 22,1\% de alunos com indicativo de sobrepeso, 14,9\% classificados com IMC $<15^{\circ} \mathrm{P}$ (indicativo de baixo peso) e 63,0\% de escolares considerados eutróficos. O resultado relativo à prevalência de sobrepeso chama a atenção, especialmente pelas conseqüências indesejáveis que a situação, se mantida, pode causar para a saúde dos alunos.

CAROBA (2002) observou ainda a prevalência 
de déficits de altura em apenas 4,0\% dos alunos, classificados com ZAI $<-2$. Note-se que a proporção identificada é o dobro da esperada $(2,0 \%)$.

KAZAPI et al. (2001), em trabalho envolvendo 797 estudantes de escolas públicas e privadas da cidade de Florianópolis - SC, também verificaram que 50\% dos adolescentes apresentaram um consumo inferior às recomendações de energia.

É importante frisar que devem ser evitadas as situações que envolvam déficit de consumo energético, pois na adolescência, como nos demais períodos de crescimento, quando a energia é limitada a proteína poderá ser usada para preenchimento das necessidades energéticas, comprometendo a velocidade de crescimento (SAITO, 1993).

ALBANO \& SOUZA (2001) concluíram que os valores médios de consumo de energia identificados por meio de pesquisa envolvendo adolescentes, ultrapassaram a recomendação, para os gêneros masculino $(136,3 \%)$ e feminino $(123,2 \%)$.

Vale enfatizar que merece cuidado o atendimento das necessidades de energia desta fase da vida, uma vez que o déficit energético poderá causar prejuízos ao crescimento e ao desenvolvimento, que se revelam bastante acelerados. Por outro lado, o número de adolescentes com sobrepeso e obesidade tem aumentado substancialmente nas últimas décadas, constituindo um importante fator de preocupação na área de saúde pública, pois estes distúrbios nutricionais, observados precocemente entre indivíduos, estão geralmente associados ao surgimento e desenvolvimento de fatores de risco que podem predispor, no futuro quando adultos, à maior incidência de distúrbios metabólicos e funcionais (MENDES et al, 2001).

Analisando-se o consumo de proteínas dos adolescentes integrantes desta pesquisa, verificou-se um valor médio de 76,6 $\mathrm{g} /$ dia (equivalente a 16,5\% do VET), praticamente alcançando a média recomendada para o grupo (10-30\% do VET), de acordo com o INSTITUTE OF MEDICINE (2002).

Estudos elaborados por ALBANO (2000), KAZAPI et al. (2001) e OLIVEIRA et al. (1998), mostraram elevado consumo protéico entre os adolescentes.
Quanto aos carboidratos (Tabela 1), verifica-se que o valor obtido foi de $229,1 \mathrm{~g} /$ dia, correspondendo a cerca de $49,3 \%$ do valor energético total da dieta. De acordo com as recomendações do INSTITUTE OF MEDICINE (2002), a ingestão diária média de carboidratos pelos adolescentes deve variar de 45 a $65 \%$ do VET.

Para os lipídios, foi observado consumo médio de $70,7 \mathrm{~g} /$ dia, o que representa $34,2 \%$ do VET, proporção que quase atinge o limite máximo da recomendação estabelecida pelo INSTITUTE OF MEDICINE (2002), que é de $25-35 \%$ do valor energético total da dieta. Tal resultado merece atenção, em decorrência das evidências que revelam associação entre elevada participação de lipídios na dieta e o favorecimento de doenças crônicas na vida adulta.

SORIANO et al. (2000), tendo por base dados de 918 estudantes espanhóis, observaram consumo elevado de lipídios nas dietas analisadas, sendo que, entre os meninos, o consumo do nutriente atingiu $33,4 \%$ do VET e, entre as meninas, alcançou 36,7\%.

FISBERG et al. (2000) recomendam consumo moderado de gorduras, principalmente as saturadas, presentes nos alimentos de origem animal. Entretanto, a dieta deverá suprir a quantidade necessária desse nutriente para o crescimento. Dietas pobres em gordura, geralmente, não fornecem a energia e os nutrientes essenciais, causando repercussões negativas para a puberdade.

É importante destacar também o reduzido consumo de fibras (13,2 $\mathrm{g} / \mathrm{dia})$, identificado entre os jovens participantes desta pesquisa (Tabela 1 ). De acordo com as recomendações do INSTITUTE OF MEDICINE (2002), a ingestão diária média de fibras de indivíduos com idade entre 14 a 18 anos deve ser de 32 g. Para a obtenção desse conteúdo, é recomendável o rotineiro consumo de frutas, vegetais, leguminosas e grãos integrais (VANNUCHI, 1990).

Segundo MACHADO \& SANTIAGO (2001), há evidências de que a presença de fibra alimentar na dieta atue na prevenção de doenças, como a diverticulose, câncer de mama e cólon, doenças coronarianas e diabetes, além de promover efeito redutor do colesterol sangüíneo.

Ainda, tendo por base os dados da Tabela 1, é 
Consumo alimentar e adolescência, Caroba et al.

Tabela 1. Conteúdo diário de energia e de nutrientes da dieta dos adolescentes. Piracicaba, 2000.

\begin{tabular}{lrcrc}
\hline & & & \multicolumn{2}{c}{ Valores } \\
\cline { 4 - 5 } Energia e Nutrientes & Média & Desvio Padrão & Mínimo & Máximo \\
\hline Energia (kcal) & 1859,1 & 758,2 & 511,2 & 4838,7 \\
Proteínas (g) & 76,6 & 35,5 & 14,2 & 260,4 \\
Carboidratos (g) & 229,1 & 104,5 & 32,1 & 671,8 \\
Lipídios totais (g) & 70,7 & 36,5 & 5,3 & 252,4 \\
Fibras (g) & 13,2 & 6,8 & 0,7 & 58,2 \\
Tiamina (mg) & 1,9 & 1,2 & 0,3 & 13,1 \\
Riboflavina (mg) & 1,6 & 1,1 & 0,2 & 18,1 \\
Niacina (mg) & 20,3 & 10,5 & 2,6 & 76,3 \\
Folacina (mg) & 167,9 & 96,1 & 20,8 & 984,2 \\
Vitamina E (mg) & 13,1 & 7,6 & 0,6 & 46,9 \\
Fósforo (mg) & 947,8 & 413,7 & 68,8 & 2715,7 \\
Ferro (mg) & 13,9 & 6,3 & 1,4 & 42,8 \\
\hline
\end{tabular}

possível notar que o consumo médio diário de tiamina (1,9 mg), riboflavina (1,6 mg) e niacina (20,3 mg) mostrou-se superior ao preconizado $(1,1 \mathrm{mg}, 1,15 \mathrm{mg}$ e $15 \mathrm{mg}$, respectivamente), enquanto os valores referentes à folacina $(167,9 \mathrm{mg})$ e à vitamina $\mathrm{E}(13,1$ $\mathrm{mg}$ ) revelaram ingestão inferior ao preconizado como referência (folacina $=400 \mathrm{mg}$ e vitamina $\mathrm{E}=$ $15 \mathrm{mg})$.

É interessante ressaltar que as vitaminas hidrossolúveis são compostos com atuação essencial em muitas etapas do metabolismo, incluindo os processos envolvendo os carboidratos, os lipídios, as proteínas e os ácidos nucléicos. De um modo geral, esses nutrientes são necessários para a manutenção, o crescimento e o funcionamento adequado do organismo (VANNUCHI \& JORDÃO JÚNIOR, 1998).

BARBIERI (1999) destaca que a vitamina E é um poderoso agente antioxidante, com capacidade de impedir a oxidação fácil dos componentes lipídicos das membranas celulares pela ação dos radicais livres. Embora as fontes naturais de vitamina E sejam amplamente disponíveis (óleos de sementes, de frutas, de vegetais e gorduras animais), o autor revela que adolescentes norte-americanos, por exemplo, ingerem exclusivamente por meio da alimentação (isto é, não foram incluídos os suplementos, etc.) quantidades insuficientes de vitamina $\mathrm{E}$.
Quanto à ingestão de fósforo pelos adolescentes, foi verificado (Tabela 1) um consumo médio (947,8 $\mathrm{mg}$ ) inferior à recomendação de $1.250 \mathrm{mg}$ (INSTITUTE OF MEDICINE, 1997).

Com relação ao fósforo, é interessante notar a expressiva proporção de adolescentes com reduzido consumo do nutriente. Ressalta-se a importância do fósforo para a dieta dos escolares, tendo em vista que o nutriente constitui aproximadamente $1 \%$ do peso corpóreo humano, estando presente principalmente na forma fosfato (DUTRA-DE-OLIVEIRA \& MARCHINI, 1998).

Quando se analisa o consumo de ferro (Tabela 1) dos escolares, verifica-se um valor médio de $13,9 \mathrm{mg} /$ dia que parece atender à recomendação (média) de ingestão deste mineral preconizada (13 mg/dia) para adolescentes (de ambos os gêneros) com idade entre 14 a 18 anos (INSTITUTE OF MEDICINE, 2001).

O ferro tem um papel fundamental na homeostase orgânica, pois participa de processos celulares vitais como: transporte de oxigênio, produção de energia por meio da síntese de ácidos nucléicos, síntese de neurotransmissores cerebrais, co-fator em reações enzimáticas e vários outros processos metabólicos (ASSAO et al, 2004). 
Consumo alimentar e adolescência, Caroba et al.

Tabela 2. Distribuição dos percentis do consumo de energia e nutrientes selecionados dos adolescentes. Piracicaba, 2000.

\begin{tabular}{|c|c|c|c|c|c|}
\hline \multirow[b]{2}{*}{ Energia e Nutrientes } & \multirow[b]{2}{*}{$10^{\circ} \mathrm{P}$} & \multirow[b]{2}{*}{$25^{\circ} \mathrm{P}$} & \multicolumn{3}{|c|}{ Valores } \\
\hline & & & $50^{\circ} \mathrm{P}$ & $75^{\circ} \mathrm{P}$ & $100^{\circ} \mathrm{P}$ \\
\hline Energia (kcal) & 991,7 & 1328,7 & 1710,5 & 2272,1 & 2916,0 \\
\hline Proteínas (g) & 36,4 & 52,8 & 71,2 & 95,9 & 121,5 \\
\hline Carboidratos (g) & 110,5 & 152,0 & 213,3 & 279,5 & 371,5 \\
\hline Lipídios (g) & 29,9 & 43,6 & 65,3 & 93,0 & 121,4 \\
\hline Fibras (g) & 5,6 & 8,8 & 12,2 & 16,5 & 21,5 \\
\hline Tiamina (mg) & 0,9 & 1,2 & 1,8 & 2,5 & 3,4 \\
\hline Riboflavina (mg) & 0,7 & 0,9 & 1,3 & 1,9 & 2,6 \\
\hline Niacina (mg) & 8,9 & 12,6 & 18,6 & 25,6 & 33,5 \\
\hline Folacina (mg) & 72,0 & 104,2 & 147,2 & 210,6 & 289,2 \\
\hline Vitamina E (mg) & 4,7 & 7,4 & 11,6 & 17,4 & 25,1 \\
\hline Fósforo (mg) & 463,7 & 663,6 & 888,3 & 1194,6 & 1479,8 \\
\hline Ferro (mg) & 7,1 & 9,4 & 12,8 & 17,1 & 21,8 \\
\hline
\end{tabular}

Cerca de 2 milhões e 150 mil crianças em idade pré-escolar são expostas ao risco para esta deficiência. O déficit de consumo pode ter reflexos no desenvolvimento mental, provocando entre outros distúrbios, apatia, irritabilidade, redução da capacidade de concentração e do aprendizado (COZZOLINO, 1999).

A seguir são apresentados os resultados da distribuição (geral) dos percentis do consumo de energia e nutrientes (Tabela 2).

Tendo por base os resultados mostrados na Tabela 2 verifica-se que os valores que separam os $10 \%$ dos escolares com o menor consumo de energia e nutrientes, não são suficientes para atender às recomendações estabelecidas para o grupo.

Inversamente, ao analisar os valores que discriminam os $10 \%$ de alunos que exibem o maior consumo de energia e nutrientes, nota-se que para energia, carboidratos, lipídios, tiamina, riboflavina, niacina, vitamina $\mathrm{E}$, fósforo e ferro esses valores superam os valores preconizados.

Atentando para a distribuição dos percentis do consumo de fibras e folacina, chama a atenção a expressiva proporção de escolares com reduzido consumo, ou seja, $90 \%$ do grupo consome quantidade inferior a 21,5g e 289,2 $\mathrm{mg}$, respectivamente. A recomendação diária para fibras é de 32g (INSTITUTE OF MEDICINE, 2002) e $400 \mathrm{mg}$ o conteúdo preconizado para a folacina (INSTITUTE OF MEDICINE, 1998); assim, é possível inferir que a maioria dos escolares apresentou déficit de consumo desses nutrientes.

Visando conhecer as distribuições dos percentis do consumo de energia e nutrientes, de acordo com o gênero, elaborou-se a Tabela 3, apresentada a seguir.

Analisando os dados relativos à distribuição do consumo de energia, é possível notar que, de forma sistemática, os valores observados são, invariavelmente, menores para o grupo das meninas.

No entanto, vale destacar que praticamente $50 \%$ das alunas revela dieta cujo conteúdo não alcança $2400 \mathrm{kcal} / \mathrm{dia}$. De acordo com FISBERG et al. (2000), há amplo acervo de registros que mostram que existem diferenças marcantes entre os gêneros, que afetam as necessidades de nutrientes e energia; por exemplo, as meninas iniciam o processo de maturação aproximadamente dois anos mais cedo do que os meninos. Os meninos experimentam maior crescimento corporal do que as meninas e possuem maior proporção de massa magra em relação ao tecido adiposo, o que condiciona maior demanda 
energética, quando comparada às necessidades do grupo feminino (FISBERG et al., 2000).

Entre os resultados relativos à proteína e aos carboidratos, deve-se enfatizar que os valores que discriminam os $10 \%$ das alunas com menor conteúdo desses nutrientes na dieta são $36,8 \mathrm{~g}(7,9 \%$ do VET) e $112,1 \mathrm{~g} \quad(24,12 \%$ do VET), respectivamente. Tais valores são ligeiramente superiores ao conteúdo de proteínas $(35,3 g-7,6 \%$ do VET) e carboidratos $(110,0 \mathrm{~g}-23,7 \%$ do VET) da dieta dos $10 \%$ dos meninos com menor consumo dos referidos nutrientes.

A Tabela 4 mostra as estimativas dos parâmetros, o respectivo teste $t$, o valor do coeficiente de determinação $\left(R^{2}\right)$ e o teste $F$ da regressão. Os testes significativos ao nível de $5 \%$ são assinalados com um asterisco. Os demais valores do teste se revelaram estatisticamente nãosignificativos.

No caso em que a variável dependente é o consumo de energia, observa-se que $c=4,73$, indicando que cada mês adicional na idade dos meninos eleva o consumo em 4,73 kcal. Mas, para as meninas esse consumo diminui $2,14 \mathrm{kcal}$ por mês (pois $4,73-6,87=-2,14)$.

O coeficiente referente à diferença de comportamento, no tocante ao consumo energético, entre os gêneros $(\mathrm{d}=-6,87)$ é estatisticamente diferente de zero, ao nível de significância de 5\%.

Ainda, de acordo com a Tabela 4, verifica-se que ao considerar a variável c (dependente), o consumo de proteínas, o valor é 0,26 . Isso indica que a cada mês acrescido à idade dos escolares, ao consumo dos adolescentes é adicionado 0,26 g de proteína, enquanto para as meninas esse consumo decresce em $0,05 \mathrm{~g}$ (obtido efetuando-se a operação: 0,26 - 0,31 = -0,05).

Também para as análises envolvendo a proteína, o coeficiente referente às diferenças de padrão de consumo, observado entre os gêneros $(d=-0,31)$, é estatisticamente diferente de zero, ao nível de significância de $5 \%$.

É possível que os resultados relativos às análises estatísticas envolvendo o consumo de energia e

Tabela 3. Distribuição dos percentis do consumo de energia e nutrientes selecionados, de acordo com o gênero dos escolares. Piracicaba, 2000

\begin{tabular}{|c|c|c|c|c|c|c|c|c|c|c|}
\hline \multirow{3}{*}{ Energia e Nutrientes } & \multicolumn{10}{|c|}{ Percentis / Gênero } \\
\hline & \multicolumn{2}{|c|}{$10^{\circ} \mathrm{P}$} & \multicolumn{2}{|c|}{$25^{\circ} \mathrm{P}$} & \multicolumn{2}{|c|}{$50^{\circ} \mathrm{P}$} & \multicolumn{2}{|c|}{$75^{\circ} \mathrm{P}$} & \multicolumn{2}{|c|}{$90^{\circ} \mathrm{P}$} \\
\hline & M & F & M & F & M & F & M & $\mathrm{F}$ & M & $\mathrm{F}$ \\
\hline Energia (kcal) & 1009,9 & 921,5 & 1364,8 & 1321,9 & 1779,0 & 1694,9 & 2324,4 & 2210,5 & 3024,4 & 2793,1 \\
\hline Proteínas (g) & 35,3 & 36,8 & 54,9 & 51,2 & 77,8 & 67,6 & 101,5 & 92,4 & 126,4 & 114,2 \\
\hline Carboidratos (g) & 110,0 & 112,1 & 149,9 & 157,2 & 219,5 & 205,6 & 295,9 & 270,6 & 397,5 & 346,6 \\
\hline Lipídios (g) & 30,3 & 29,9 & 43,8 & 43,6 & 66,7 & 63,9 & 94,4 & 88,2 & 121,6 & 119,7 \\
\hline Fibras (g) & 6,0 & 5,6 & 9,3 & 8,5 & 12,5 & 12,0 & 17,4 & 15,9 & 22,6 & 20,4 \\
\hline Tiamina (mg) & 0,9 & 0,8 & 1,3 & 1,2 & 1,9 & 1,7 & 2,7 & 2,4 & 3,53 & 3,2 \\
\hline Riboflavina (mg) & 0,7 & 0,6 & 1,0 & 0,9 & 1,4 & 1,3 & 2,1 & 1,8 & 2,9 & 2,3 \\
\hline Niacina (mg) & 9,2 & 8,8 & 13,3 & 12,2 & 19,3 & 17,7 & 27,8 & 23,1 & 36,8 & 30,7 \\
\hline Folacina (mg) & 74,6 & 71,0 & 102,9 & 106,6 & 145,0 & 150,7 & 210,1 & 210,6 & 288,3 & 295,3 \\
\hline Vitamina E (mg) & 5,2 & 4,7 & 7,9 & 7,0 & 12,3 & 10,9 & 17,5 & 17,1 & 23,6 & 23,0 \\
\hline Fósforo (mg) & 491,9 & 455,5 & 690,9 & 636,1 & 919,2 & 861,2 & 1216,1 & 1140,1 & 1603,1 & 1423,9 \\
\hline Ferro (mg) & 7,6 & 6,7 & 10,5 & 8,8 & 13,6 & 11,9 & 18,6 & 16,0 & 24,1 & 19,9 \\
\hline
\end{tabular}


Tabela 4. Equações de regressão estimadas - consumo de energia e nutrientes selecionados em função da idade e gênero dos adolescentes. Piracicaba, 2000.

\begin{tabular}{|c|c|c|c|c|c|c|}
\hline \multirow[t]{2}{*}{ Energia e Nutrientes } & \multicolumn{4}{|c|}{ Coeficiente } & \multirow[b]{2}{*}{$R^{2}$} & \multirow[b]{2}{*}{$F$} \\
\hline & $\mathrm{a}$ & $\mathrm{b}$ & $\mathrm{c}$ & $\mathrm{d}$ & & \\
\hline \multirow[t]{2}{*}{ Energia (kcal) } & 1141 & 1008 & 4,73 & $-6,87$ & 0,015 & $2,79^{(*)}$ \\
\hline & $(2,98)$ & $(1,90)$ & $(2,05)$ & $(-2,12)$ & & \\
\hline \multirow[t]{2}{*}{ Proteínas (g) } & 37,21 & 43,25 & 0,26 & $-0,31$ & 0,023 & $4,41^{(*)}$ \\
\hline & $(2,09)$ & $(1,75)$ & $(2,46)$ & $(-2,07)$ & & \\
\hline \multirow[t]{2}{*}{ Carboidratos (g) } & 161,95 & 87,36 & 0,44 & $-0,60$ & 0,0068 & 1,26 \\
\hline & $(3,07)$ & $(1,19)$ & $(1,39)$ & $(-1,35)$ & & \\
\hline \multirow[t]{2}{*}{ Lipídios (g) } & 43,53 & 44,54 & 0,18 & $-0,30$ & 0,0102 & 1,89 \\
\hline & $(2,37)$ & $(1,74)$ & $(1,61)$ & $(-1,91)$ & & \\
\hline \multirow[t]{2}{*}{ Fibras $(\mathrm{g})$} & 18,74 & $-1,15$ & $-0,03$ & 0,00029 & 0,014 & 2,61 \\
\hline & $(5,51)$ & $(-0,24)$ & $(-1,49)$ & $(0,01)$ & & \\
\hline \multirow[t]{2}{*}{ Tiamina (mg) } & 1,35 & 0,46 & 0,0047 & $-0,0044$ & 0,015 & 2,80 \\
\hline & $(2,27)$ & $(0,56)$ & $(1,31)$ & $(-0,87)$ & & \\
\hline \multirow[t]{2}{*}{ Riboflavina (mg) } & 0,67 & 0,052 & 0,0061 & $-0,0016$ & 0,018 & 3,42 \\
\hline & $(1,18)$ & $(0,07)$ & $(1,78)$ & $(-0,34)$ & & \\
\hline \multirow[t]{2}{*}{ Niacina (mg) } & 12,14 & 9,6 & 0,057 & $-0,075$ & 0,022 & 4,14 \\
\hline & $(2,31)$ & $(1,31)$ & $(1,81)$ & $(-1,67)$ & & \\
\hline \multirow[t]{2}{*}{ Folacina (mg) } & 114,24 & $-6,07$ & 0,32 & 0,062 & 0,0055 & 1,02 \\
\hline & $(2,36)$ & $(-0,09)$ & $(1,08)$ & $(0,15)$ & & \\
\hline \multirow[t]{2}{*}{ Vitamina E (mg) } & 12,53 & 7,53 & 0,0056 & $-0,050$ & 0,0088 & 1,64 \\
\hline & $(3,28)$ & $(1,42)$ & $(0,24)$ & $(-1,55)$ & & \\
\hline \multirow[t]{2}{*}{ Fósforo (mg) } & 590,73 & 419,86 & 2,41 & $-3,03$ & 0,0156 & 2,93 \\
\hline & $(2,84)$ & $(1,45)$ & $(1,92)$ & $(-1,72)$ & & \\
\hline \multirow[t]{2}{*}{ Ferro (mg) } & 13,18 & 2,39 & 0,011 & $-0,027$ & 0,0285 & 5,42 \\
\hline & $(4,17)$ & $(0,54)$ & $(0,56)$ & $(-1,02)$ & & \\
\hline
\end{tabular}

${ }^{(*)}=$ nível de significância de $5 \%$. 
proteína revelem um comportamento, mais evidente entre as meninas, de maior preocupação, especialmente com os aspectos estéticos relacionados ao peso corporal, em parte decorrente do intenso processo de exposição às informações veiculadas pela mídia, que valorizam padrões de beleza relacionados invariavelmente ao baixo Índice de Massa Corporal -IMC.

Cabe lembrar que provavelmente as meninas reduzem o consumo energético e também protéico sem maiores preocupações e cuidados com a ingestão de vários nutrientes considerados essenciais neste estágio de vida. Em decorrência desse comportamento comprometem de maneira expressiva as chances de adotarem dietas balanceadas.

Examinando os resultados obtidos para os demais nutrientes selecionados, verifica-se que os coeficientes referentes à diferença de comportamento entre os gêneros não são estatisticamente significativos (Tabela $4)$.

É interessante frisar que pesquisa divulgada pelo INSTITUTO SODEXHO PARA O DESENVOLVIMENTO DA QUALIDADE DE VIDA NO COTIDIANO (2001) revelou alguns resultados interessantes sobre consumo alimentar de crianças e jovens com idade entre 5 e 17 anos. As informações da pesquisa foram obtidas junto aos institutos internacionais, centros de pesquisa e por meio da realização de entrevistas com especialistas de vários países. Foram analisados dados coletados em países como Bélgica, Brasil, Canadá, França, Alemanha, Itália, Holanda, Espanha, Suécia, Reino Unido e Estados Unidos.

No conjunto dos países integrantes do referido estudo, a quantidade de energia consumida cotidianamente pelas crianças e pelos jovens revela decréscimo nas últimas décadas. Mais recentemente, esta tendência parece acentuar a diferença do consumo energético entre os meninos e as meninas. No ano de 2000, o consumo energético médio diário dos meninos superou em $55,8 \%$ a ingestão de energia identificada entre as meninas (INSTITUTO SODEXHO..., 2001).

Outro dado interessante da referida pesquisa é que o consumo de energia, tendo por base o gênero, também varia entre os grupos de crianças de distintos países. Em lugares onde a comida é mais elaborada, as meninas tendem a limitar esse consumo de maneira ainda mais expressiva. Nesse sentido, a diferença na ingestão de energia entre meninos e meninas é maior na França $(69 \%)$ e menor no Canadá (21\%).

Vale registrar que, tendo em vista a estrutura socioeconômica da maioria dos países, especialmente aqueles classificados como desenvolvidos, onde foram obtidos e analisados os dados pelos responsáveis pela pesquisa, é provável que as diferenças encontradas, entre os gêneros, envolvam um conjunto maior de nutrientes, em relação à amostra representativa da população jovem brasileira.

No entanto, ao examinar os resultados mostrados na Tabela 4 fica evidente que, por meio da adoção da técnica da regressão múltipla, foi possível captar, entre os escolares da rede pública de ensino de Piracicaba, diferenças de comportamentos revelados por meninos e meninas, exclusivamente no tocante ao consumo de energia.

Segundo ALBANO \& SOUZA (2001), na adolescência, a maior ingestão de alimentos com elevado conteúdo de energia coincide com o pico da velocidade máxima do crescimento, e pode ser observado real aumento do apetite relacionado ao adequado atendimento das necessidades. Existem variações expressivas entre os gêneros, sendo que os meninos ingerem, a cada idade, quantidades maiores de alimentos que fornecem maior conteúdo de energia em relação às meninas.

\section{Conclusão}

Em média, os adolescentes participantes da pesquisa apresentaram um consumo de energia inferior ao preconizado, sendo que os valores observados são, invariavelmente, menores para os grupos das meninas.

É possível notar também que, em cada mês adicional na vida dos meninos, há um aumento do consumo de 4,73 kcal e de 0,26 g de proteínas, enquanto que para as meninas o consumo energético diminui 2,14 kcal e o de proteínas $0,05 \mathrm{~g}$ por mês.

Para os demais nutrientes, verifica-se que os coeficientes referentes à diferença de comportamento entre os gêneros não são estatisticamente significativos.

$\mathrm{Na}$ adolescência o indivíduo adquire comportamentos que, em grande parte, serão 
mantidos ao longo da vida. Sendo assim, esse é o momento privilegiado para as intervenções na área da saúde e da nutrição, tendo em vista a adoção de hábitos de vida saudáveis e a promoção da saúde na vida adulta.

Pode-se inferir que a saúde é um conceito abstrato para os indivíduos muito jovens e a orientação nutricional deve se basear em fundamentos concretos, enfatizando os alimentos, na forma de cardápios e sugestões, entre outras, para substituições prazerosas, criativas e nutritivas. As mudanças devem ser estimuladas, sem traumas e imposições, a fim de promover e assegurar o bom estado de saúde.

Deve-se salientar que os estudos para monitoramento do consumo alimentar de adolescentes devem ser contínuos, tendo em vista sua importância para subsidiar, entre outras atividades, a elaboração e implementação de programas na área de saúde, envolvendo a prevenção de carências nutricionais.

\section{Referências Bibliográficas}

ALBANO, R.D. Estado nutricional e consumo alimentar de adolescentes. São Paulo, 2000. Dissertação (Mestrado) - Faculdade de Saúde Pública, Universidade de São Paulo.

ALBANO, R.D.; SOUZA, S.B. Ingestão de energia e nutrientes por adolescentes de uma escola pública. Jornal de Pediatria, Porto Alegre, v. 77, n. 6, p. 512516, 2001.

ALBUQUERQUE, M.F.M.; MONTEIRO, A.M. Ingestão de alimentos e adequação de nutrientes no final da infância. Revista de Nutrição, Campinas, v. 15, n. 3, p. 291-299, 2002.

ASSAO, T.Y. et al. A importância do ferro na saúde e nutrição do grupo materno-infantil. Compacta Nutrição, São Paulo, v. 5, n. 3, p. 7-22, 2004.

BARBIERI, D. Micronutrientes em pediatria. In: De Angelis R.C. Fome oculta, impacto para a população do Brasil. São Paulo: Atheneu, p.88-93, 1999.

CAROBA, D.C.R. A escola e o consumo alimentar de adolescentes matriculados na rede pública de ensino. Piracicaba, 2002, 162 p. Dissertação (Mestrado) - Escola Superior de Agricultura "Luiz de Queiroz", Universidade de São Paulo.

CAVALCANTE, A.A.M.; PRIORE， S.E.; FRANCESCHINI, S.C.C. Estudos de consumo alimentar: aspectos metodológicos gerais e seu emprego na avaliação de crianças e adolescentes. Rev. Bras. Saúde Materno Infantil, Recife, v. 4, n. 3, p. 229-240, 2004.

COZZOLINO, S.M.F. Fortificação de alimentos versus biodisponibilidade. In: De Angelis R.C. Fome oculta, impacto para a população do Brasil. São Paulo: Atheneu, p.143-154, 1999.

DUTRA-DE-OLIVEIRA, J.E.; MARCHINI, J.S. Macrominerais. In: Dutra-de-Oliveira, J.E., Marchini, J.S. Ciências nutricionais. São Paulo: Sarvier, p.133165, 1998.

FISBERG, M. et al. Hábitos alimentares na adolescência. Pediatria Moderna, São Paulo, v. 36, n. 11, p. 724-734, 2000.

FRANCISCHI, R.P.P. et al. Obesidade: atualização sobre sua etiologia, morbidade e tratamento. Revista de Nutrição, Campinas, v. 13, n. 1, p. 17-28, 2000.

INSTITUTE OF MEDICINE. Dietary Reference Intakes for Calcium, Phosphorus, Magnesium, Vitamin D, and Fluoride. Food and Nutrition Board. Washington, DC: National Academy Press, 1997.

Dietary Reference Intakes for Thiamin, Riboflavin, Niacin, Vitamin B6, Folate, Vitamin B12, Pantothenic Acid, Biotin, and Choline. Food and Nutrition Board. Washington, DC: National Academy Press, 1998.

Dietary Reference Intakes for Vitamin C, Vitamin E, Selenium, and Carotenoids. Food and Nutrition Board. Washington, DC: National Academy Press, 2000.

Dietary Reference Intakes for Vitamin A, Vitamin K, Arsenic, Boron, Chromium, Copper, Iodine, Iron, Manganese, Molybdenum, Nickel, Silicon, Vanadium, and Zinc. Food and Nutrition Board. Washington, DC: National Academy Press, 2001. 
Dietary Reference

Intakes for Energy, Carbohydrate, Fiber, Fat, Fatty Acids, Cholesterol, Protein and Amino Acids. Food and Nutrition Board. Washington, DC: National Academy Press, 2002.

INSTITUTO BRASILEIRO DE GEOGRAFIA E ESTATÍSTICA. Fundo das Nações Unidas para a Infância. Indicadores sobre crianças e adolescentes. Brasília: UNICEF/IBGE, 242p, 2001.

Censo 2000. Disponível em: $<$ http://www.ibge.gov.br/ $/>$. Acesso em: 02 mar 2005.

INSTITUTO SODEXHO PARA O DESENVOLVIMENTO DA QUALIDADE DE VIDA NO COTIDIANO. Masculino e feminino: os diferentes hábitos alimentares entre crianças e jovens. São Paulo: Sodexho Alliance, 29p, 2001.

KAUFMANN, A. Transtornos alimentares na adolescência. Revista Brasileira de Medicina, n.1, out. 2000. Disponível em: <http:// www.cibersaude.com.br/search.asp >. Acesso em: 05 mai 2002.

KAZAPI, I.M. et al Consumo de energia e macronutrientes por adolescentes de escolas públicas e privadas. Revista de Nutrição, Campinas, v. 14 (Supl.), p. 27-33, 2001.

MACHADO, F.M.S.; SANTIAGO, V.R. O papel das frutas e hortaliças na nutrição humana. In: Torres, E.A.F.S., Machado, F.M.S. Alimentos em questão: uma abordagem técnica para as dúvidas mais comuns. São Paulo: Ponto Crítico, p.25-34, 2001.

MENDES, F.S.V. et al. Avaliação do estilo de vida e condições nutricionais de adolescentes atendidos em um programa específico. Nutrição em Pauta, São Paulo, v. 9, n. 47, p. 20-24, 2001.

OLIVEIRA, J. et al. A alimentação dos escolares no município de São Paulo. In: Anais do Congresso Brasileiro de Ciência e Tecnologia de Alimentos, Rio de Janeiro: SBCTA, p.495-498, 1998.

PHILIPPI, S.T.; SZARFARC, S.C.; LATTERZA, A.R. Virtual Nutri [software]. Versão 1.0. Universidade de São Paulo, Faculdade de Saúde Pública, Departamento de Nutrição, 1996.

SAITO, I.M. Nutrição. In: Coates, V., Françoso, L.A., Beznos, G.W. Medicina do adolescente. São Paulo: Sarvier, p.37-50, 1993.

SAS INSTITUTE. The SAS System [software]. Version 8. Cary, 1998.

SORIANO, J.M.; MOLTÓ, J.C.; MAÑES, J. Dietary intake and food pattern among university students. Nutrition Research, New York, v. 20, n. 9, p. 1249$1258,2000$.

VANNUCHI, H.; JORDÃO JÚNIOR, A.A. Vitaminas hidrossolúveis. In: Dutra-de-Oliveira, J.E., Marchini, J.S. Ciências nutricionais. São Paulo: Sarvier, p.191-207, 1998.

VANNUCHI, H. Aplicações das recomendações nutricionais adaptadas à população brasileira. Cadernos de Nutrição, São Paulo, v. 2, 156p, 1990.

VILLAR, B.S. Desenvolvimento e validação de um questionário semi-quantitativo de freqüência alimentar para adolescentes. São Paulo: 2001. Tese (Doutorado) - Faculdade de Saúde Pública, Universidade de São Paulo. 